\title{
An Early Diagnosis of Endocarditis Facilitated by the Electronic Stethoscope
}

\author{
Walid Barake, Amer M. Johri* \\ Cardiology Division, Queen's University, Kingston, Canada \\ Email: ${ }^{*}$ amerschedule@gmail.com
}

Received 14 April 2014; revised 14 May 2014; accepted 13 June 2014

Copyright (C) 2014 by authors and Scientific Research Publishing Inc.

This work is licensed under the Creative Commons Attribution International License (CC BY).

http://creativecommons.org/licenses/by/4.0/

(c) (i)

\section{Abstract}

The practice of cardiac auscultation is a critical tool used by physicians to detect alterations in the cardiovascular system. A case of both left and right sided endocarditis initially detected by electronic auscultation in a woman with a history of injection drug use is described. The electronic stethoscope, with the ability to amplify heart sounds, established the presence of both a systolic and diastolic murmur when standard auscultation failed to detect the diastolic component. Urgent standard echocardiography confirmed concurrent tricuspid and aortic valves endocarditis, and the patient was referred for surgical evaluation urgently. The present case demonstrates the value of the electronic stethoscope to amplify murmurs in the early detection of endocarditis. The case presented also serves as a useful reminder that right-sided endocarditis can have important leftsided complications.

\section{Keywords}

Electronic Stethoscope, Conventional Stethoscope, Endocarditis, Murmurs

\section{Introduction}

Most patients with significant valvular heart disease are first diagnosed based on the finding of a murmur [1] Previous studies have suggested that the electronic stethoscope is significantly better in detecting low frequency sounds, is safe to be used in telemedical referral of patients with heart murmurs and has its place in everyday bedside practice [2] [3]. A comparison of the electronic stethoscope and the conventional auscultation has not been reported in the context of infective endocarditis.

\section{Case Report}

A 45-year-old woman with a history of injection drug use presented to the Emergency Department (ED) with Corresponding author. 
fever, chills and productive cough. Her past medical history was significant for epidural abscess (T2 - T6) (Methicillin sensitive Staphylococcus aureus in blood and epidural abscess) diagnosed 6 months prior and treated with decompressive laminectomy and intravenous (IV) antibiotics. The patient had no known history of valve abnormality as evidenced by a normal transthoracic echocardiogram (TTE) and transesophageal echocardiogram (TEE) during her previous admission to neurosurgery.

On physical examination, the patient was febrile $\left(39^{\circ} \mathrm{C}\right)$ and was found to have a blood pressure of $97 / 46$ $\mathrm{mmHg}$. She had a heart rate of 107 beats/min, a respiratory rate of 20 breaths $/ \mathrm{min}$ and pulse oximetry of $97 \%$ on room air. Her jugular venous pulsation was elevated and she was found to have a grade 2/6 pansystolic murmur, loudest at the tricuspid area, which had not been previously documented. No other murmur was detected by a conventional stethoscope. Using an electronic stethoscope (3 M Littmann, model $3200 \mathrm{TM}$ ) an early blowing diastolic murmur was detected on the left parasternal area third intercostal space using maximum amplification (Sound 1).

A third year medical student, a senior internal medicine resident (W.B) and an experienced Cardiologist listened carefully with their conventional cardiac stethoscopes to the patient using the appropriate sitting up and breath holding technique and were not able to detect the diastolic murmur. The rest of the physical exam was significant for Janeway lesions on the patient's palms and soles (Figure 1). A hand-held bedside ultrasound (Vscan, GE Healthcare) was performed and revealed signs of tricuspid regurgitation with possible vegetation attached to the valve. However, the aortic valve was not fully visualized due to artefact from breast implants. Laboratory investigations revealed a white blood cell count of $13.9 \times 10^{9} / \mathrm{L}$ (normal range $4.0 \times 10^{9} / \mathrm{L}$ to $10.5 \times$ $10^{9} / \mathrm{L}$ ) and a hemoglobin level of $98 \mathrm{~g} / \mathrm{L}$ (normal range $120 \mathrm{~g} / \mathrm{L}$ to $160 \mathrm{~g} / \mathrm{L}$ ). Her creatinine was 40 (normal range $<80 \mu \mathrm{mol} / \mathrm{L}$ ).

She was treated with IV ampicillin and gentamicin. Based on the electronic stethoscope findings, a STAT TTE was ordered instead of an urgent (same day) one and confirmed the suspicion of both right and left sided endocarditis. It showed a mobile mass on the ventricular aspect of the non-coronary cusp of the aortic valve $(0.5$

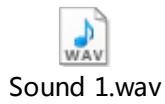

Sound 1. Auscultation recording over the left parasternal area $3^{\text {rd }}$ intercostal (20 fold amplification). A pan-systolic as well as decrescendo diastolic murmur became more apparent when the patient held her breath at 00:20. A head phone or loud speaker should be used to obtain high sound quality from this recording.

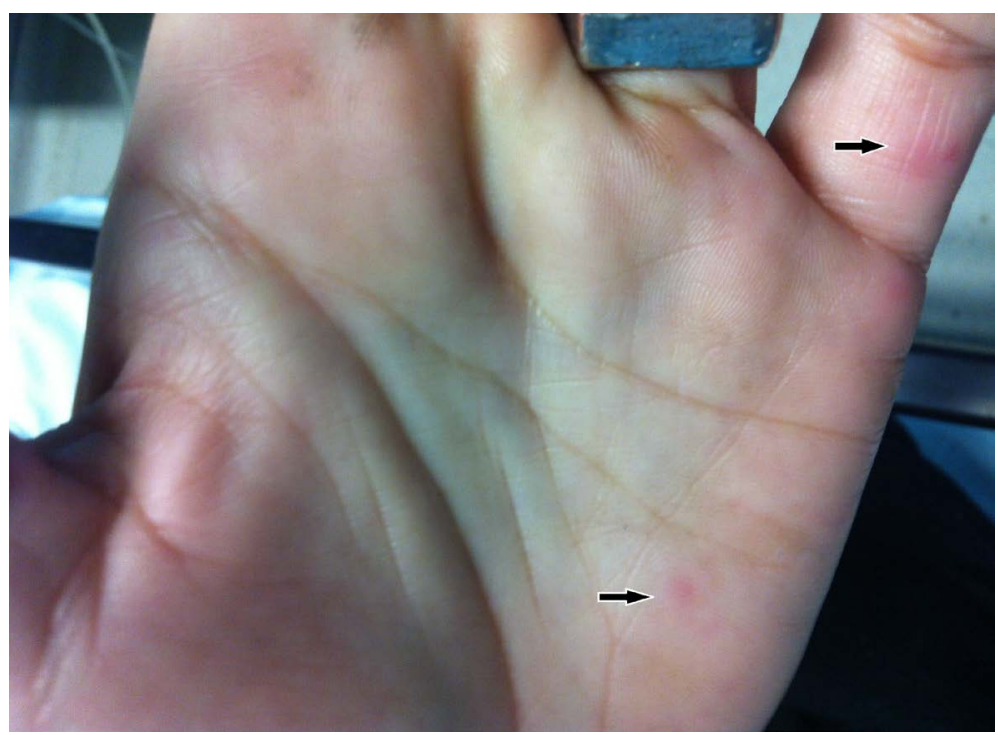

Figure 1. Non-tender, small erythematous nodular lesions on the left palm and little finger suggestive of Janeway lesions (arrows). 
$\times 1.5 \mathrm{~cm}$ ). The mass was associated with moderate aortic regurgitation. Additionally, there was a mobile mass on the tricuspid valve $(0.8 \times 1.7 \mathrm{~cm})$ with associated moderate to severe tricuspid regurgitation. Left and right ventricular size and systolic function were normal. The patient was referred to Cardiac surgery urgently and underwent aortic valve replacement with tricuspid valve repair. Following repair, the patient recovered well and was discharged home following a 4-week course of IV antibiotics. The use of the electronic stethoscope in this case led to an earlier diagnosis and referral to cardiac surgery and potentially improved the clinical outcome.

\section{Discussion}

The practice of cardiac auscultation is a vital component of the cardiac physical exam, alerting the physician to alterations in the cardiovascular system. Most patients with significant valvular heart disease are first diagnosed based upon the finding of a murmur. Auscultation has a reported sensitivity of 70 percent and a specificity of 98 percent for the detection of valvular heart disease [1]. However, the sensitivity and specificity vary substantially with the expertise of the examiner and probably with the instrument used.

Discovery of a diastolic murmur is essential for the diagnosis of aortic regurgitation. In a review of the literature, the presence of an early diastolic murmur was the most useful finding for establishing the presence of aortic regurgitation (positive likelihood ratio 8.8 [i.e., the odds of aortic regurgitation are increased 8.8-fold]) and its absence the most useful finding for eliminating the presence of aortic regurgitation (negative likelihood ratio 0.2 to 0.3 [i.e., the odds of disease are reduced by a factor of 0.2 to 0.3 ]) [4]. The murmur of aortic regurgitation is best heard with the diaphragm of the stethoscope. Low-intensity, high-pitched aortic regurgitation murmurs may not be heard unless firm pressure is applied with the diaphragm of the stethoscope over the left sternal border or over the right second interspace, while the patient sits and leans forward with the breath held in full expiration [4].

The diagnosis of infective endocarditis (IE) is usually based upon a combination of factors, including both a history and physical examination; the physical examination should include a careful cardiac examination for signs of new regurgitant murmurs or heart failure. A thorough search should be undertaken for the classic clinical stigmata of endocarditis, including evidence of small and large emboli with special attention to the fundi, conjunctiva, and skin. The revised Duke criteria remains the most commonly accepted diagnostic criteria for IE [5].

To the best of our knowledge, a formal comparison of the electronic stethoscope and traditional auscultation has not been reported in the context of infective endocarditis. In 1996, Lukin A. et al. [2] compared two stethoscopes, the classic acoustic stethoscope (Littmann 2120) and an electronic one with the sound amplifier and the noise filtering system (Medmax2) in 10 patients examined by 10 physicians. Significantly better detection of low frequency sounds was found in favour of the electronic stethoscope (chi $2=17.9$; $p<0.0001$ ). It was concluded that the selective amplification improved the stethoscope performance and has its place in everyday bedside practice, especially in departments of cardiology.

Dahl L.B. et al. [3] assessed the clinical utility of recording heart murmurs in children by electronic stethoscopes and then emailing the recordings for remote assessment by Cardiologists. Heart sounds from 47 patients with no murmur $(n=7)$, with innocent murmurs $(n=20)$, or with pathological murmurs $(n=20)$ were recorded using a sensor based stethoscope and e-mailed to a remote computer. Four Cardiologists assessed and categorised the cases as having "no murmur", "innocent murmur", or "pathological murmur", recorded the assessment time per case, their degree of certainty, and whether they recommended referral. The mean sensitivity and specificity were $89.7 \%$ and $98.2 \%$ respectively. A total of $93.4 \%$ of cases with a pathological murmur and $12.6 \%$ of cases with an innocent murmur were recommended for referral. It was concluded that telemedical referral of patients with heart murmurs for remote assessment by a Cardiologist is safe and saves time [3].

\section{Conclusion}

Some limitations of the electronic stethoscope are apparent. The electronic stethoscopes require a functioning battery to auscultate properly which can pose problems. Referring to the $3 \mathrm{M}^{\mathrm{TM}}$ Littmann ${ }^{\circledR}$ Model 3200 manual, the battery will last for approximately 60 hours of continuous use [6]. Before the battery is depleted completely, a warning tone will indicate that the battery has only two more hours of continuous use. Sound quality remains constant even on low battery [6]. Other issues involve cost and maintenance above and beyond what is required for the simple traditional stethoscope. As the traditional stethoscope is ubiquitous, some clinicians remain con- 
cerned about the loss of traditional auscultation skills if new trainees rely heavily on the electronic stethoscope and occasionally find themselves without access to this technology in unforeseen circumstances (such as battery depletion). As with the advent of many new medical technologies in this era, the potential to improve bedside care is exciting, but requires a considered approach to implement. Hence, more research is needed to better define the utility and limitations of this new technology.

\section{Declaration of Interest}

No financial support was received for the preparation of this manuscript and authors have no relationships with industry to disclose. No Potential conflicts of interest to disclose.

\section{References}

[1] Mineo, K., Cummings, J., Josephson, R. and Nanda, N.C. (2001) Acquired Left Ventricular Outflow Tract Obstruction during Acute Myocardial Infarction: Diagnosis of a New Cardiac Murmur. The American Journal of Geriatric Cardiology, 10, 283-285. http://dx.doi.org/10.1111/j.1076-7460.2001.00038.x

[2] Lukin, A., Polić, S., Rumboldt, Z., Bagatin, J., Rakić, D. and Kuzmanić, A. (1996) Comparison of Auscultation Findings Using a Classic Stethoscope (Litmann 2120) and Electronically Amplifiedstethoscope (Medmax2). Lijec Vjesn, 118, $127-128$.

[3] Dahl, L.B., Hasvold, P., Arild, E. and Hasvold, T. (2002) Heart Murmurs Recorded by a Sensor Based Electronic Stethoscope and e-Mailed for Remote Assessment. Archives of Disease in Childhood, 87, 297-301. http://dx.doi.org/10.1136/adc.87.4.297

[4] Choudhry, N.K. and Etchells, E.E. (1999) The Rational Clinical Examination. Does This Patient Have Aortic Regurgitation? JAMA, 281, 2231-2238. http://dx.doi.org/10.1001/jama.281.23.2231

[5] Durack, D.T., Lukes, A.S. and Bright, D.K. (1994) New Criteria for Diagnosis of Infective Endocarditis: Utilization of Specific Echocardiographic Findings. American Journal of Medicine, 96, 200-209. http://dx.doi.org/10.1016/0002-9343(94)90143-0

[6] (2013) $3 \mathrm{M}^{\mathrm{TM}}$ Littmann ${ }^{\circledR}$ Stethoscopes > Service \& Support > FAQs. http:/www.littmann.com/wps/portal/3M/en US/3M-Littmann/stethoscope/customer-service/help/ 